\title{
¿EXISTE UN LEVANTAMIENTO DEL VELO JURISDICCIONAL?: EL LEVANTAMIENTO DEL VELO SOCIETARIO COMO EXTENSIÓN DEL CONVENIO ARBITRAL Y COMO REMEDIO DE FONDO
}

IS THERE A PIERCING OF THE JURISDICTIONAL VEIL? THE PIERCING OF THE CORPORATE VEIL AS AN EXTENSION OF THE ARBITRATION AGREEMENT TO NON-SIGNATORIES AND AS A REMEDY FOR THE MERITS

\author{
Alejandra Quintanilla Gutiérrez* \\ Exmiembro del Consejo Ejecutivo de THËMIS-Revista de Derecho \\ Instituto Nacional de Defensa de la Competencia y \\ de la Protección de la Propiedad Intelectual
}

\begin{abstract}
In arbitration matters, one of the cases in which the effects of the arbitration agreement can be extended to a non-signatory party is through the piercing of corporate veil doctrine. The doctrine justifies the existence of such theory in order to include into the arbitration process parent or related companies that use subsidiaries as a shell to commit abuses and defraud their creditors. Moreover, corporate doctrine, no matter the jurisdictional system, establishes that the piercing of corporate veil is a remedy that applies only when the defensive separation of assets is broken and liability is assigned to a parent o related company.

In this article, the author exposes the confusion related to arbitration matters in the application of the piercing of the corporate veil as a nonsignatory doctrine and as a substantial remedy. Thus, it proposes a harmonization of the figure, in order to stablish a comprehensive application.
\end{abstract}

KEYWORDS: Arbitration; non-signatory parties; piercing of the corporate veil; limited liability.
En materia arbitral, uno de los supuestos en el que los efectos del convenio arbitral pueden extenderse a una parte no signataria es mediante la doctrina del levantamiento del velo societario. La doctrina justifica la existencia de dicha teoría a fin de incluir en el arbitraje empresas matrices o vinculadas que utilizan cascarones societarios para cometer abusos y defraudar acreedores. Por otro lado, la doctrina societaria, independientemente del sistema jurisdiccional, establece que el levantamiento del velo es un remedio que solo se configura cuando se rompe la separación defensiva de activos y se asigna responsabilidad a un tercero vinculado.

En el presente artículo, la autora expone la confusión existente en materia arbitral en la aplicación del levantamiento del velo societario como supuesto de extensión del convenio y como remedio de fondo. Así, propone una armonización de la figura, a fin de establecer una aplicación integral.

PALABRAS CLAVE: Arbitraje; partes no signatarias; levantamiento del velo societario; responsabilidad limitada.

\footnotetext{
Bachiller en Derecho por la Pontificia Universidad Católica del Perú (PUCP). Asistente legal corporativa en la Comisión de Defensa de la Libre Competencia del Indecopi (Lima, Perú). Contacto: alejandra.sqg@gmail.com.

La autora agradece a Nicolás Hernández Bernal por su guía y paciencia en la redacción de este artículo.

Nota del Editor: El presente artículo fue recibido por el Consejo Ejecutivo de THĒMIS-Revista de Derecho el 20 de mayo de 2020, y aceptado por el mismo el 26 de junio de 2020.
} 


\section{INTRODUCCIÓN}

En el arbitraje, existen principios fundamentales que permiten identificar quienes son las partes sujetas al convenio arbitral y quienes no se encuentran comprendidos dentro de dicha relación jurídica. Asumiendo la naturaleza consensual de esta institución ${ }^{1}$, el principio de inevitabilidad del arbitraje entiende a las partes firmantes de un contrato, el cual contiene una cláusula arbitral, como las únicas con la capacidad de participar en el proceso. Es por ello que, en la mayoría de casos, si se desea determinar a las partes bajo el convenio, solo es necesario observar la hoja de firmas (Born, 2009, p. 1136).

No obstante, existen casos excepcionales en los cuales se pretende justificar la extensión subjetiva de la cláusula arbitral a una parte no signataria, en razón de su comportamiento y vinculación con el contrato, o dada su relación con las partes suscriptoras. La solicitud de incorporar a una de esas partes al arbitraje crea una tensión entre dos principios fundamentales. Por un lado, se desea mantener la naturaleza consensual del convenio arbitral, restringiendo el vínculo jurídico a las partes que expresaron su voluntad; y, por otro lado, maximizar la efectividad práctica del laudo arbitral vinculando a las personas relacionadas (sean naturales o jurídicas) ${ }^{2}$.

A fin de poder solucionar la tensión entre dichos principios, la doctrina arbitral ha generado una variedad de supuestos jurídicos para vincular a partes no signatarias del convenio arbitral. Por ejemplo, la teoría de la agencia (actual y aparente), la teoría del consentimiento implícito, grupo de compañías, estoppel, tercero beneficiario, levantamiento del velo societario, garante, subrogación, sucesión legal, entre otras (Born, 2009, p. 1223). Como podemos denotar de la nomenclatura de dichas teorías, vincular a partes no signatarias al arbitraje ha requerido la aplicación de principios del derecho comercial, corporativo y en general del derecho de los contratos (Born, 2009, p. 1224). Una de estas teorías, creada sobre la base de principios del derecho corporativo y que será objeto de análisis en el presente artículo, es la teoría del levantamiento del velo societario.

Ahora bien, esta doctrina se caracteriza por buscar extender la jurisdicción arbitral a aquellas entidades que, escudadas bajo el principio de responsabilidad limitada de las personas jurídicas, pretenden evitar las consecuencias legales de conductas indebidas o fraudulentas que han realizado mediante el uso de empresas subordinadas o vinculadas (Born, 2014, p. 1431). La terminología del levantamiento del velo societario en el arbitraje como presupuesto de extensión del pacto arbitral se inspiró en el remedio del mismo nombre creado jurisprudencialmente en el siglo XIX por las cortes judiciales de tradición del common law. En ese sentido, su evolución ha permitido que hoy sea tomada en cuenta tanto en cortes judiciales del sistema continental como en el arbitraje.

En relación con este último aspecto, se tiene que tomar en cuenta que ambas teorías se aplican en el arbitraje: (i) la del levantamiento del velo para extensión del convenio arbitral; y, (ii) así como la del levantamiento del velo como remedio jurídico. Asimismo, persiguen el mismo fin -esto es, la relativización del principio de responsabilidad limitada y separación de activos de las empresas para sancionar el uso de la figura societaria como forma de defraudación de acreedores. En consecuencia, surgen varias preguntas: ¿realmente estamos ante dos figuras con nombres iguales, pero con sustanciales diferencias? ¿Puede entenderse como una sola figura en el arbitraje, pero con un análisis en dos etapas o fases? ¿Es posible seguir llamando "levantamiento del velo societario" a una figura que no está relacionada con la responsabilidad limitada?

A fin de dar luz a dichas interrogantes, en un primer momento, se expondrá con mayor detalle los antecedentes y para uniformizar la terminología se precisarán los conceptos del levantamiento del velo societario en el arbitraje y como remedio de orígenes judiciales. En un segundo momento, se expondrá la confusión existente en materia ar-

Sobre este aspecto, Díaz-Candia ha mencionado que

[l]a principal disyuntiva que ha enfrentado el arbitraje como institución en Latinoamérica es la de su propia naturaleza jurídica. El debate parece centrarse entre dos extremos: uno contractualista y otro jurisdiccionalista. Para el extremo contractualista, el arbitraje es un mecanismo en el cual las partes de un contrato de alguna forma insertan a terceros (los árbitros y, en ocasiones, un centro de arbitraje) en dicho contrato, delegando su voluntad o consentimiento para que esos terceros puedan injerir en la ejecución o terminación del contrato (2015, p. 124).

2 Sobre este aspecto, el autor Park ha mencionado lo siguiente:

[f]or arbitrators, motions to join non-signatories create a tension between two principles: maintaining arbitration's consensual nature, and maximizing an award's practical effectiveness by binding related persons. Pushed to the limit of their logic, each goal points in an opposite direction. Resolving the tension usually implicates the two doctrines discussed below: implied consent and disregard of corporate personality $(2009$, p. 3) 
bitral en la aplicación del levantamiento del velo societario como supuesto de extensión del convenio y como remedio de fondo. Así, se evidenciará como se suele diluir el análisis de la figura jurisdiccional con la sustancial, evaluando para cuestiones de extensión del convenio el elemento del fraude. Es decir, se suele establecer que no se le debe extender el convenio arbitral a una parte no signataria sin antes (o, por defecto, a su vez) asignarle responsabilidad.

Finalmente, se propondrá a la comunidad arbitral la necesidad de abordar ambas figuras de forma separada en los procedimientos arbitrales. Ahora bien, pese a buscar el mismo fin, el análisis que un tribunal debe realizar al momento de decidir extender o no el convenio arbitral a uno no signatario, debe ser sustancialmente distinto al análisis que se realice para determinar la responsabilidad de ese no signatario. La idea anterior posee el objeto de evitar cuestionamientos a la decisión de extensión del convenio arbitral relacionadas a supuestos adelantamientos de opinión sobre el fondo de la controversia en una etapa en la que no corresponde.

\section{PRECISIONES CONCEPTUALES}

Antes de un análisis crítico sobre las figuras del levantamiento del velo societario tanto en la doctrina arbitral como en la doctrina societaria, es necesario realizar algunas precisiones conceptuales. Dichas precisiones tienen el fin de aclarar las diferencias en el origen de las figuras estudiadas y exponer sus principales antecedentes. En ese sentido, se hará una breve mención sobre los antecedentes y evolución de las figuras del levantamiento del velo para: (i) la doctrina societaria (y su utilidad para la asignación de responsabilidad); y, (ii) la doctrina arbitral (como un supuesto de extensión del convenio arbitral a partes no signatarias).

\section{A. La doctrina del levantamiento del velo so- cietario como remedio de origen judicial}

Desde el siglo XIX, un principio fundamental del derecho societario ha sido que los accionistas, en cualquier compañía, no responden por las obligaciones que esa última pueda adquirir más allá del capital que han invertido. En efecto, este principio fundamental responde a la responsabilidad limitada. Dicha figura tiene innumerables ventajas como reducción de los costos de agencia, incentivo de manejo eficiente por parte de los representantes, establecimiento de un valor exacto de las compa- ñías, entre otras (Garcia-Gallont \& Kilpinen, 2015, pp. 1232-1233).

Ahora bien, la representación de la responsabilidad limitada se suele dar por una defensa. Dicha defensa permite que los accionistas de una sociedad sean responsables solo por el monto que invirtieron y no por las obligaciones de la sociedad en sí misma. En efecto, detrás de la responsabilidad limitada se encuentra la existencia de la separación defensiva de activos de la corporación. Este aspecto, en palabras de Hansmann y Kraakman significa que "los acreedores de la empresa no tienen ningún derecho en absoluto sobre los activos personales de los accionistas de la empresa, que sirven exclusivamente para dar seguridad a los acreedores personales de dichos accionistas" (2003, p. 19).

Sin embargo, no todas las consecuencias de la responsabilidad son tan beneficiosas. Cuando las empresas no pueden satisfacer todos sus créditos, dependiendo del orden de prelación de pago de los acreedores, algunos de estos quedarán con sus créditos impagos. Esto genera una ventaja para los accionistas y para las empresas que forman parte de un grupo económico y, en algunos casos, esa ventaja es usada para realizar actos de defraudación.

En base a este último aspecto, en el siglo XIX, surgió la siguiente pregunta: ¿cómo se puede hacer justicia ante este uso fraudulento de las sociedades? La respuesta formulada por los jueces ingleses y americanos fue que la responsabilidad no puede ser absoluta y existen casos excepcionales en donde se puede hacer responsable a personas fuera del alcance de la compañía a través de diversos métodos. Uno de esos métodos es el levantamiento del velo societario.

Uno de los primeros antecedentes fue el emblemático caso inglés Salomon c. Salomon \& Company Limited, en el año $1897^{3}$. En este caso la Corte de Apelaciones declaró que la compañía era un mito y que Salomon (responsable de esta misma) había constituido una compañía en contra de la verdadera intención de la Ley de Sociedades Inglesa de 1862. En ese sentido, se concluyó que la sociedad había llevado a cabo un crédito como agente de Salomon, quien debería, por lo tanto, ser responsable de la deuda incurrida. No obstante, dicha sentencia fue revocada por la Cámara de los Lores, quienes dejaron fuera los elementos subjetivos de la incorporación de los accionistas al pacto social.

3 Para mayor información, véase la página web de Legal Information Institute de Cornell Law School (https://www.law. cornell.edu/wex/piercing_the_corporate_veil). 
Al ser un remedio de origen jurisdiccional, la doctrina del levantamiento del velo societario se encuentra en permanente construcción. Esta característica ha llevado a innumerables artículos en la doctrina estadounidense sobre el establecimiento de un orden en los criterios de los jueces para no convertir la figura en una herramienta impredecible y desordenada que atiende a los fines personales de los jueces.

Actualmente, el caso Walkovszky c. Carlon es parte de la jurisprudencia líder sobre levantamiento del velo societario. En dicho caso, la Corte de Apelaciones de Nueva York sostuvo que un demandante necesita probar que un accionista usó a la compañía como su agente para realizar negocios a título individual. Solo se podrá levantar el velo cuando las pruebas determinen que la compañía es un agente de su accionista y se responsabilizará a los accionistas debido a la doctrina del respondeat superior ${ }^{4}$. En otros estados americanos (como Florida y Texas) se necesita probar necesariamente indicios como, por ejemplo, el uso de la compañía como un alter ego de la matriz o sus accionistas; y, además, que dicha matriz o los accionistas estén coludidos en la acción fraudulenta 5 .

No obstante, aun cuando la ley societaria en los Estados Unidos se basa, de forma principal, en la ley estatal (por ello, cada estado tiene sus propios precedentes vinculantes), prácticamente todas las jurisdicciones estatales se suscriben a una de las dos herramientas tradicionales de jurisprudencia de levantamiento del velo. Estos son los tres factores que incluyen la doctrina de instrumentalidad y la doctrina de alter ego ${ }^{6}$. Ahora bien, la jurisprudencia tiene claro que, a fin de poder aplicar el remedio del levantamiento del velo societario, se tendrá que aplicar alguna teoría que grafique cuál es la vinculación fraudulenta entre la compañía y las personas (naturales o jurídicas) relacionadas (Cheng-Han et al., 2019, p. 150).

Es así que si se encuentran indicios de una persona que usa el control de la compañía para promover su propio negocio en lugar del negocio de la corporación, con la consecuencia de que la corporación era solo un "caparazón", se podría utilizar la doctrina del alter ego para poder levantar el velo societario de una compañía (Cheng-Han et al., 2019 p. 152).

No obstante, existiendo diversas teorías para usar el remedio del levantamiento del velo societario, Macey y Mitts consideran que los factores que las cortes americanas han utilizado para justificar el levantamiento del velo societario son similares (2014, p. 108). En efecto, dichos autores listaron los indicios más importantes de la siguiente manera:

- Subcapitalización significativa de la compañía (los requisitos de capitalización varían según la industria, la ubicación y las circunstancias de la empresa).

- Incumplimiento de observar las formalidades corporativas en términos de comportamiento y documentación.

- Mezcla de actividades o activos de la compañía y de los accionistas.

- Los accionistas tratan a los activos de la compañía como suyos.

- $\quad$ Incumplimiento en el pago de dividendos.

- Desvío de fondos corporativos por el accionista o los accionistas dominantes mediante, por ejemplo, pago irregular de dividendos.

- Oficinas corporativas sin funcionamiento.

- $\quad$ Ausencia o inexactitud de los registros corporativos.

- $\quad$ Superposición de registros corporativos, funciones y personal.

- Uso de la compañía como una "fachada" a fin que los accionistas dominantes puedan utilizar sus fondos para temas personales. Este indicio es conocido como la teoría del alter ego.

- Uso de la compañía para promover fraude, injusticia o actividades ilegales.

4 Para mayor información, véase la página web de Legal Information Institute de Cornell Law School (https://www.law. cornell.edu/wex/piercing_the_corporate_veil).

5 Para mayor información, véase la página web de Legal Information Institute de Cornell Law School (https://www.law. cornell.edu/wex/piercing_the_corporate_veil).

$6 \quad$ En ese sentido, en el caso RRX Indus. Inc. c. Lab-Con. Inc., la Corte declaró que la doctrina del alter ego se aplica cuando (1) existe tal unidad de interés y propiedad que las personalidades de la corporación y el individuo ya no están separadas, y (2) se producirá un resultado desigual si los actos se tratan solo como los de la corporación (Cheng-Han et al., 2019, p. 152) 
La compañía asume el pago de obligaciones individuales de accionistas dominantes o actores relacionados ${ }^{7}$.

Es necesario precisar que cada uno de estos factores debe ser analizado en el contexto especifico de la controversia. De hecho, sería absurdo asignar responsabilidad a un accionista alegando que la compañía ha incumplido observar formalidades corporativas o ha registrado información corporativa de forma inexacta. Es necesario generar la conexión entre el daño del demandante con las conductas ilícitas que provocan el no pago de un crédito.

Ahora bien, con respecto a la jurisprudencia nacional, en la sentencia del 5 de marzo de 2013, recaída en el Expediente No. 451-2009, emitida por la Primera Sala Civil con Subespecialidad Comercial de la Corte Superior de Justicia, donde se establece lo siguiente:

La teoría del levantamiento del velo societario no es una figura extraña a nuestro sistema jurídico. Es una figura novedosa que ha tenido desarrollo doctrinario y, como tal, su aplicación no genera una irregularidad. Desde el momento en que hablamos de la aplicación de la teoría del levantamiento del velo societario, no podemos afirmar que existe desconocimiento de categorías como la responsabilidad limitada o la personalidad jurídica [...]. Dentro del contenido constitucionalmente protegido del derecho a la libertad de la empresa [...] no se encuentra el beneficio de la responsabilidad limitada ni de la personalidad jurídica de las sociedades anónimas. Mucho menos la extensión constitucional cuando esas categorías se utilizan con la finalidad de cometer fraude (2013, p. 16).

Los jueces reconocen de manera poco clara que el sistema jurídico no puede amparar el uso de categorías jurídicas para cometer fraude. Es por ello que limita la responsabilidad limitada y reconoce que el levantamiento del velo no desconoce dicho principio, pero este mismo no debe estar considerado dentro del contenido constitucionalmente protegido del derecho a la libertad de empresa.

En conclusión, el remedio del levantamiento del velo societario desde esta perspectiva es un remedio proveniente del common law, de construcción jurisprudencial. Para poder aplicar dicho remedio a un caso concreto, las cortes estadounidenses utilizan diversas doctrinas, dependiendo de la relación y los indicios existentes entre la compañía y sus accionistas.

En ese sentido, la doctrina de la instrumentalidad o del alter ego son aplicadas en los casos en donde las pruebas indican que una de las compañías fue utilizada como un cascarón o instrumento. En otros casos, la doctrina de la agencia se utiliza en algunos estados cuando los fines de constitución de una compañía no son meramente instrumentales, pero dicho fin fraudulento fue construyéndose a través del tiempo.

\section{B. La doctrina del levantamiento del velo so- cietario como supuesto de extensión del convenio arbitral a partes no signatarias}

Uno de los supuestos de extensión del pacto arbitral a partes no signatarias se configura mediante la llamada doctrina del levantamiento del velo societario o del alter ego. Dicha doctrina se caracteriza por buscar extender la jurisdicción arbitral a aquellas entidades que, escudadas bajo el principio de responsabilidad limitada de las personas jurídicas, pretenden evitar las consecuencias legales de conductas indebidas o fraudulentas que han realizado mediante el uso de empresas subordinadas o vinculadas (Born, 2014, p. 1431).

Ahora bien, dependiendo la jurisdicción, se han empleado diferentes terminologías para referirse a la doctrina del alter ego $o^{8}$. No obstante, la característica común de todas ellas es la relativización de la responsabilidad limitada de las personas jurídicas para evitar abusos por parte de corporaciones que buscan defraudar a sus acreedores. Por otro lado, con bastante frecuencia se confunde esta teoría con la referida al grupo de sociedades. Sin embargo, en la doctrina del levantamiento del velo societario se le da relevancia al fraude o el abuso de derecho de una forma corporativa para limitar la responsabilidad de la parte real. Por el contrario, en la doctrina del grupo de sociedades se le da relevancia especial a la intención de cada una de las empresas de un grupo para derivar una intención para arbitrar (Besson, 2010, p. 149).

7 Macey y Mitts resaltan el fracaso de las cortes americanas para crear un vínculo lógico entre los factores encontrados en las pruebas y los objetivos de políticas públicas. En ese sentido, mencionan lo siguiente:

[f]or example, while it often is claimed that piercing the corporate veil is required to prevent fraud, this is not the case. Where there is fraud, a cause of action for fraud can be brought directly, and there is no reason to pierce the corporate veil in order to prevent injustice. Similarly, where there is no fraud, it hardly makes sense to impose liability as a means of preventing some other, unrelated fraud in the future (2014, pp. 108).

8 Así lo ha expresado Born, quien establece, por ejemplo, como en alemán esta doctrina es conocida como durchgriff, en francés como levée du voile social, en español como levantamiento del velo societario y en muchos otros contextos del idioma inglés como piercing (perforar) o lifting (levantar) el corporate veil (velo corporativo) (2014, p. 1432). 
En ese sentido, autores como Born resaltan las diferencias entre las dos teorías expuestas de la siguiente manera:

La teoría del alter ego es una regla de derecho que se invoca para ignorar o anular los efectos aplicables de la incorporación de la personalidad jurídica separada. El resultado de este análisis es que una entidad se considera inexistente o simplemente una parte no incorporada de otra entidad. [...]. Por el contrario, la doctrina del grupo de empresas suele ser un medio para identificar las intenciones de las partes, lo que no perturba ni afecta la personalidad jurídica de las entidades en cuestión (2009, p. 1432).

Queda claro que la doctrina del levantamiento del velo corporativo pertenece al derecho de sociedades, mientras que la doctrina del grupo de empresas es predominantemente una teoría del derecho contractual (Cohen, 2008, p. 23). Dicha pertenencia impacta de forma importante en las normas aplicables a cada una. Ahora bien, autores como Caivano precisan que la sociedad puede ser desestimada cuando se encuentra formada con propósitos fraudulentos o impropios.

Se establece, entonces, una lista de supuestos que los jueces norteamericanos han predicado a fin de levantar el velo de una sociedad (Caivano, 2006, p. 134):

- La existencia de un fraude o completo dominio de la sociedad controlante que lleve a confusión a los terceros.

- Conductas o circunstancias que impliquen un virtual abandono de la separación de sus identidades societarias. Dichas conductas son dos de forma específica: (i) la subsidiaria no posee cuentas bancarias, oficinas, ni papelería, no realiza transacciones ni tiene actividad; o, (ii) la controlante y la subsidiaria comparten oficinas y personal, tienen los mismos directores, mezclan fondos y no se consideran como unidades de ganancia separadas $^{9}$.

Por otro lado, Ferrario establece que la doctrina del levantamiento del velo societario en el arbitraje señala que este tiene tres diferentes vertientes: (i) doctrina de la instrumentalidad; (ii) la doctrina del alter ego; y, (iii) la doctrina de la identidad. (2009, pp. 655-657). Procedemos, por lo tanto, a desarrollar brevemente las vertientes mencionadas.

En primer lugar, con respecto a la doctrina de la instrumentalidad, se distinguen supuestos de aplicación cuando: (i) existe un excesivo ejercicio de control de los accionistas con respecto a la compañía; (ii) se presenta una conducta ilícita o desigual; $y$, (iii) se genera una relación de causalidad con respecto a la pérdida económica del demandante. En ese sentido, se debe de comprobar que la compañía ha sido creada como un instrumento para la defraudación.

En segundo lugar, bajo la doctrina del alter ego, se deben cumplir dos presupuestos para su consideración: (i) una fuerte unidad de propiedad e intereses entre la matriz y la subsidiaria, de tal forma que los límites entre ambas sean borrosos y casi inexistentes; y, (ii) el elemento del fraude o abuso de derecho. Así, en el caso Van Dorn Corporation c. Future Chemical \& Oil Corporation, el Tribunal de Apelaciones del Sétimo Circuito resaltó lo siguiente:

A corporation is a legal entity separate and distinct from its shareholders, directors and officers, and, generally, from other corporations with which it may be affiliated. [...] however, a corporate entity will be disregarded and the veil of limited liability pierced when two requirements are met: (i) first, there must be such a unity of interest and ownership that the separate personalities of the corporation and the individual [or other corporation] no longer exist; and, (ii) second, circumstances must be such that adherence to the fiction of separate corporate existence would sanction a fraud or promote injustice (citado en Ferrario, 2009, pp. 660-661).

En tercer lugar, la doctrina de la identidad es la vertiente menos importante del levantamiento del velo societario. Bajo esta misma, la identidad corporativa separada de la subsidiaria (que no se considera como un agente de la matriz) no se tiene en cuenta y, como consecuencia, tanto ella como la corporación matriz se consideran y tratan como una sola compañía.

Con respecto a la aplicación de la doctrina a un caso concreto, Bullard González establece que es necesaria la presencia de dos requisitos: el elemento objetivo y el elemento subjetivo. El elemento objetivo es la existencia de control de la matriz a la subsidiaria, o de los accionistas a la compañía.

$9 \quad$ Para mayor información, véase a Caivano (2006), quien resalta que dichos supuestos han sido clasificados a partir de la siguiente jurisprudencia: Tribunal de Apelaciones del Segundo Circuito (in re Passalacqua Builders), Tribunal de Apelaciones del Segundo Circuito (in re Carte Blanche), y Tribunal de Apelaciones del Segundo Circuito (in re Walter E. Heller \& Co. c. Video Innovations Inc.). 
El elemento subjetivo, por su parte, es la voluntad de fraude común a todos los actores involucrados (2008, pp. 224-225).

Ahora bien, es importante resaltar que el Decreto Legislativo 1071, Decreto Legislativo que norma el arbitraje (en adelante, Ley de Arbitraje), es uno de los pocos productos normativos que establece la extensión del convenio arbitral a partes no signatarias de forma expresa. En efecto, sobre el artículo 14 de la Ley de Arbitraje ${ }^{10}$, podemos comprender que

no registra paralelismos en otras legislaciones extranjeras, constituye por lo mismo una de las innovaciones más importantes [...] y tiene una consecuencia práctica enorme pues establece criterios expresos mediante los cuales es posible concebir como partes del convenio arbitral a personas o entidades que no han suscrito el contrato que lo contiene (Conejero Roos \& Irra de la Cruz, 2012, p. 57).

La Ley de Arbitraje contempla diversos criterios de extensión de la jurisdicción arbitral, los cuales pueden ser utilizados para aplicar la doctrina del levantamiento del velo societario. Si nos remitimos al artículo 14 de la norma, podemos identificar como criterio principal que la participación activa o la intención de derivar beneficios del contrato pueden ser utilizados para analizar la conducta de un no signatario.

Con base a lo anteriormente expuesto, podemos identificar que bajo la doctrina del levantamiento del velo societario se confunde una finalidad tanto jurisdiccional como de fondo. En otras palabras, en un primer momento se extiende la jurisdicción arbitral a efectos de que una parte no signataria participe en el arbitraje y de forma posterior se evalúa si este no signatario es responsable jurídicamente por las conductas alegadas en el fondo.

\section{ANÁLISIS COMPARATIVO DEL LEVANTA- MIENTO DEL VELO EN LA DOCTRINA ARBI- TRAL Y SOCIETARIA EN CONJUNTO CON LA NECESIDAD DE LA DIFERENCIACIÓN ENTRE AMBAS}

De las precisiones conceptuales del tratamiento del levantamiento del velo societario en el arbitraje y como remedio de origen judicial, en conjunto a los criterios de aplicación establecidos tanto por autores de la doctrina societaria como autores especialistas en arbitraje, se concluye que la figura responde a diferentes necesidades de las dos vías jurisdiccionales. No obstante, ambas cuentan con un fin común: la asignación de responsabilidad a partes que injustamente utilizan la responsabilidad limitada para defraudar acreedores.

Con respecto al levantamiento del velo como remedio, este se aplica como consecuencia de descubrir actos fraudulentos con respecto los accionistas o alguna matriz. La causa puede ser cualquiera de las teorías que las cortes judiciales han motivado para justificar la aplicación del remedio (alter ego, instrumentalidad, agencia, responsabilidad vicaria, entre otras). Este remedio genera que los créditos sean satisfechos por la persona (natural o jurídica) que controla las decisiones de la compañía demandada.

Asimismo, la competencia de los jueces se asume de acuerdo a la normativa aplicable y se materializa en la aplicación del remedio como mecanismo de protección de los derechos de crédito. En efecto, cuando inicia el juicio, la parte demandante inicia un proceso contra la compañía y sus accionistas, quienes han pasado por un examen de legitimidad. Es en el propio juicio que se analizan dos aspectos. Por una parte, la unidad entre los accionistas y la compañía; por otra, si se generase un resultado desigual si los actos realizados solo se adjudican a la compañía, los cuales de no ser remediados generarían que el sistema de justicia permitiese actos fraudulentos (Cheng-Han et al., 2019, p. 152).

Ahora bien, en caso se logre superar el estándar de prueba judicial, se aplicaría el levantamiento del velo societario a fin que los accionistas o la matriz respondan por el fraude a los acreedores. En el arbitraje, los árbitros deben respetar la naturaleza consensual del convenio arbitral, por lo que existen importantes impedimentos para aplicar un remedio jurídico a partes no signatarias. Es por este último aspecto que la doctrina del levantamiento del velo societario pretende cumplir una finalidad tanto jurisdiccional como de fondo.

\section{A. Planteamiento del problema}

Si se toman en cuenta ambos enfoques, resulta confuso referirse nominalmente al levantamiento del

10 Dicha norma menciona lo siguiente:

El convenio arbitral se extiende a aquellos cuyo consentimiento de someterse a arbitraje, según la buena fe, se determina por su participación activa y de manera determinante en la negociación, celebración, ejecución o terminación del contrato que comprende el convenio arbitral o al que el convenio esté relacionado. Se extiende también a quienes pretendan derivar derechos o beneficios del contrato, según sus términos (Decreto Legislativo 1071, 2008). 
velo como un supuesto de extensión del convenio arbitral sin que, de forma necesaria, este tenga en cuenta el quebrantamiento de la responsabilidad limitada. A nivel judicial, no existe la necesidad imperante de justificar la competencia del juez a fin de traer a juicio a alguna empresa del grupo o, a su vez, a los propios accionistas. En cambio, en el arbitraje es necesario realizar la extensión del convenio para que de forma posterior los árbitros puedan asignar responsabilidad a una parte no signataria del convenio arbitral. Sin dicha extensión el levantamiento del velo como remedio no podría aplicarse.

En ese sentido, Palacios Pareja establece que "sí es posible que el árbitro desgarre el velo societario, evaluando si existe o no un grupo económico, de manera de resolver en el laudo que empresas lo conforman y, por consiguiente, extendiéndoles el alcance de su decisión" (2008, p. 171). El propósito del levantamiento del velo es el de tomar en cuenta las relaciones que existen detrás de una compañía sin haber utilizado verbos relacionados a una consecuencia jurídica, como si solo se pretendiera descubrir quiénes son las personas que se encuentran detrás de una compañía, controlándola y utilizándola como una protección para sus acciones. En ese sentido, el saber quiénes se encuentran detrás de la compañía no es "rasgar" el velo societario; sin embargo, es un paso fundamental para aplicar el remedio jurídico.

En ese sentido, el problema planteado deviene en que el levantamiento del velo es un remedio cuya aplicación es necesariamente de fondo por el elemento del fraude. El mismo remedio es considerado por la doctrina arbitral como un elemento jurisdiccional para extender el convenio a no signatarios. Sin embargo, en el ámbito arbitral se presenta como complicación que no se puede determinar que al demandado no signatario se le debe extender el convenio arbitral sin antes (o, a su vez) asignarle responsabilidad. Para que se pueda configurar la extensión del convenio por la teoría del levantamiento del velo se requiere probar, por lo menos, un fraude o propósito indebido.

Las oportunidades en las cuales puede presentarse la solicitud de la incorporación de un no signatario son diversas. Estas pueden darse con la petición arbitral, la demanda, la contestación o con una solicitud de incorporación en la etapa postulatoria. De presentarse solicitudes de extensión del convenio y excepciones de competencia, los árbitros están facultados por la Ley de Arbitraje a emitir los laudos parciales para poder decidir sobre, por ejemplo, controversias de jurisdicción.

En la medida que un tribunal arbitral puede emitir un laudo parcial para resolver las excepciones de jurisdicción, no figurarían problemas en que se aplique de manera independiente la extensión del convenio arbitral por el levantamiento del velo societario. Esto debido a que el análisis de fondo se restringiría al último aspecto: establecer remedios a las partes que ya fueron definidas en el laudo parcial.

Sin embargo, en la práctica, la complejidad del análisis de los indicios del levantamiento del velo hace que su aplicación también se dificulte. En efecto, si los árbitros son quienes deciden la incorporación de terceros no signatarios antes de analizar los elementos de prueba (interrogatorios, pericias que establezcan o no la vinculación, pruebas documentales o testimoniales), puede que se pronuncien teniendo un análisis incompleto. No obstante, ¿̇la lógica anterior es correcta?

B. ¿Es el supuesto de extensión del convenio arbitral una aplicación de la doctrina del levantamiento del velo societario como remedio?

De acuerdo a lo establecido por De Trazegnies Granda,

una parte no suscriptora debe ser sometida a la obligación de arbitrar cuando la otra parte pretende razonablemente que su conducta ha sido fraudulenta con el objeto de confundir a la parte demandante o de colaborar en eludir la responsabilidad de la demandada suscriptora (2004, p. 19).

Una de las causas de extensión del convenio es la superación de un estándar de razonabilidad. De acuerdo a dicha postura, si deseamos que el convenio arbitral alcance a un tercero no firmante, es necesario que ya se hayan explorado diversos indicios que permitan razonablemente creer que estamos ante un grupo económico con voluntad fraudulenta.

Dicho estándar de razonabilidad fue mencionado por el tribunal arbitral conformado para resolver la controversia suscitada entre TSG Perú S.A.C., como demandante, contra Pesquera Chicama S.A.C., Langostinera Caleta Dorada S.A.C, Pesquera Libertad S.A.C., Procesadora del Campo S.A.C. y Pesquera Industrial Katamarán S.A.C. En dicha controversia, se estableció que

[el levantamiento del velo] únicamente será posible en tanto el material probatorio disponible indique que se han cumplido dos presupuestos fundamentales para dicha aplicación. (i) una probada vinculación entre las empresas involucradas; y, (ii) una voluntad de fraude de dichas empresas en relación a la supuesta afectada (citado en Repetto et al., 2014, p. 2020). 
De acuerdo con el tribunal, el análisis de los elementos objetivos y subjetivos del caso que permitirán aplicar el supuesto de levantamiento del velo para extender el convenio, forman parte del análisis jurisdiccional (o de forma) del caso. Sin embargo, dichos elementos son indispensables para fundamentar una decisión sobre el fondo de la controversia que implique la asignación de responsabilidad a un tercero no firmante del convenio. La determinación de la vinculación empresarial y el ánimo de defraudar de dicho grupo son algunos de los indicios listados como factores o indicadores de una controversia que requiere la aplicación del levantamiento del velo societario como remedio jurídico.

Por lo tanto, los elementos que ayudan a una aplicación de la doctrina del levantamiento del velo societario como supuesto de extensión del convenio arbitral y, a su vez, los indicios utilizados para aplicar el remedio del levantamiento del velo societario como evasión de la separación defensiva de activos y asignación del crédito a un tercero parte del grupo económico, son casi los mismos.

Estamos, por tal motivo, frente a un nuevo dilema del huevo y la gallina. En efecto, se realiza un estándar de razonabilidad para justificar la extensión del convenio que contiene un análisis sobre el fraude, el cual resulta necesario para concluir la asignación de responsabilidad a terceros no signatarios. Así pues, ¿cuál fue primero? ¿asignar responsabilidad para extender o extender para asignar responsabilidad?

Es importante resaltar que, a la luz de la doctrina societaria, la aplicación del levantamiento del velo societario como remedio significa que el débito (el cual se encuentra en cabeza de la sociedad) deba ser satisfecho por cualquier otra persona (natural o jurídica) vinculada a ella y que haya realizado actos de fraude utilizando a la sociedad como una fachada, un instrumento o una agencia para la celebración de negocios jurídicos. En ese sentido, utilizar el término "rasgar" el velo societario para describir el acceso a la información sobre la conformación del grupo económico haría referencia a los pasos previos necesarios a fin de justificar la aplicación del remedio.

El acceso a la información del grupo económico y la forma en la cual este se vincula es un paso necesario para que (al final del proceso) se rompa con la separación de activos, se ignore la responsabilidad limitada de la sociedad afiliada del inversor o de otro tercero vinculado y se pueda exigir la disposición de su esfera patrimonial a fin de responder a un crédito que, en principio, no era suyo. Ahora bien, surge la siguiente pregunta: ¿ंes el supuesto de extensión del convenio arbitral una aplicación de la doctrina del levantamiento del velo societario como remedio? La respuesta es un rotundo no y creer lo contrario sería confundir dos figuras diferentes.

En efecto, ninguno de los autores analizados considera que ese primer paso sea la aplicación de la doctrina del levantamiento del velo tal y como la doctrina societaria la describe. Todos concluyen que la asignación de responsabilidad a una empresa que forma parte del grupo económico es el objetivo final de todo el proceso y que la extensión del convenio arbitral en dichas circunstancias es un paso indispensable para la aplicación del remedio.

En ese sentido, la línea divisoria entre el análisis de competencia y el análisis de fondo en el arbitraje es muy tenue. Como se explicó antes, la decisión con respecto a la competencia del árbitro en las pretensiones sometidas a controversia ante un arbitraje (sobre todo, cuando se tratan de partes no signatarias) se suele realizar junto con el análisis de fondo de la controversia. Esto debido a que los árbitros podría considerar contingente tomar una decisión sobre la extensión sin haber analizado las pruebas suficientes. Dicha decisión adelanta un análisis de fondo, todavía cuando solo sea resolver una excepción de competencia.

En el análisis de jurisdicción no se vulnera la responsabilidad limitada de ninguna de las partes intervinientes; sino que solo se resuelve la propia jurisdicción del tribunal arbitral. Es por ello que, dadas las necesidades de los procesos arbitrales, el levantamiento del velo como extensión tuvo que configurarse.

\section{Análisis de otros supuestos de extensión del convenio arbitral para aplicar el reme- dio del levantamiento del velo societario}

Ahora bien, podría argumentarse que es innecesario entender al levantamiento del velo societario como un procedimiento para armonizar los campos de aplicación de la doctrina, en la medida que, para aplicar el levantamiento del velo como remedio, no es estrictamente necesario utilizar el levantamiento del velo como supuesto de extensión; puesto que pueden existir otros que cumplen con los mismos fines. En ese caso, surge la siguiente pregunta: ¿se puede utilizar cualquiera de las diversas doctrinas establecidas para traer a una parte no signataria al arbitraje, independientemente de si la decisión de fondo tendrá como remedio el levantamiento del velo societario? Hagamos el ejercicio con otro supuesto de extensión del convenio arbitral: el grupo de sociedades.

De acuerdo con Ferrario, para la aplicación de la doctrina del grupo de empresas, ni la voluntad de 
fraude ni el vínculo causal con el perjuicio al acreedor son requisitos de aplicación considerados por la jurisprudencia (2009, p. 669). Algunos de los elementos generalmente identificados por la doctrina para analizar la existencia del grupo societario son los siguientes: (i) la intención de todas las partes involucradas de considerar a todo el grupo como parte contratante, independientemente de cuál fue la empresa que suscribe o ejecuta el contrato; y, (ii) la participación de los no signatarios en la negociación, desempeño o terminación del contrato. Ello demuestra la voluntad de las compañías del grupo de ser parte del contrato $y$, como consecuencia, ser parte del convenio arbitral a pesar de que no lo hayan firmado.

Se deduce, por lo tanto, que incluso el elemento de control que se encuentra estrictamente relacionado con la existencia de un grupo de empresas no tendrá la misma relevancia y peso que tendrá en relación con el levantamiento del velo societario (Ferrario, 2009, pp. 669-670). En ese sentido, este mismo concluye con lo siguiente:

[p]arece claro que las dos doctrinas (el grupo de sociedades y el levantamiento del velo) están basadas en diferentes condiciones [...]. El levantamiento del velo societario tiene como objetivo evitar la injusticia que se genera al no ignorar los principios de separación y responsabilidad limitada de la compañía misma. La doctrina del grupo de empresas tiene, en cambio, un objetivo diferente, que está relacionado con los efectos del acuerdo de arbitraje. El efecto directo de un acuerdo válido es conferir competencia a los árbitros que decidirán la disputa entre las partes. El acuerdo de arbitraje, por lo tanto, no solo prueba que las partes dieron su consentimiento para presentar sus disputas ante un tribunal arbitral en lugar de los tribunales nacionales, también constituye la fuente básica de los poderes y autoridad del mismo $^{11}$ (Ferrario, 2009, p. 670) [el énfasis es nuestro] [traducción libre].

De acuerdo a lo mencionado, no es posible reemplazar la doctrina del grupo de sociedades por la doctrina del levantamiento del velo societario porque la propia extensión del convenio arbitral tiene el mismo fin que el análisis de fondo: evitar la injusticia que se produce cuando se abusa de la figura de la responsabilidad limitada. La extensión forma parte del propio procedimiento para probar la voluntad de fraude del grupo económico, por lo tanto, análisis de jurisdicción y fondo se encuentran totalmente vinculados.

\section{Aplicación de la extensión del convenio sin la aplicación del remedio: ¿es posible?}

¿Es posible que existan casos en donde un tribunal arbitral se haya declarado competente para decidir sobre las pretensiones que involucran a partes no signatarias, pero no se haya utilizado el remedio del levantamiento del velo societario? Park considera que sí y, con respecto a la cuestión presentada, menciona lo siguiente:

[s]e puede invocar el levantamiento del velo para justificar la jurisdicción [del tribunal arbitral] sobre una filial corporativa o la responsabilidad de una compañía por las deudas sustantivas de otra. Esto no significa, sin embargo, que los árbitros deban (o estén obligados) a encontrar al accionista [o filial] responsable de las obligaciones de la subsidiaria [...]. Aunque ambas compañías hayan acordado someterse al mismo procedimiento arbitral, el árbitro podría determinar que ninguna de las compañías es responsable de las obligaciones de la otra ${ }^{12}$ $(2009$, p. 6) [traducción libre].

En ese sentido, si seguimos considerando una visión restringida de la doctrina del levantamiento

\section{Traducción libre:}

In light of the above, it therefore seems clear that the two doctrines in question are based on different conditions. The difference in relation to the conditions is connected to and reflects the different purposes underlying these two doctrines. The piercing or lifting the corporate veil doctrine aims to avoid the injustice arising from not disregarding the two fundamental principles of separateness and limited liability of the companies of the same group. Hence, piercing the corporate veil is allowed in order to prevent an inequitable outcome. The group of companies' doctrine has, instead, a different objective, which is related to the effects of the arbitration agreement. The direct effect of a valid arbitration agreement is to confer jurisdiction on the arbitrators to decide the dispute between the parties. The arbitration agreement, therefore, does not only prove that the parties consented to submit their disputes to arbitration rather than national courts, but it also constitutes the main and basic source of the powers and authority of the arbitration tribunal (Ferrario, 2009, p. 670) [el énfasis es nuestro].

12 Traducción libre:

Veil-piercing may be invoked to justify either jurisdiction over a corporate affiliate or one company's liability for the substantive debts of another. This does not mean, however, that arbitrators who join a non-signatory parent must (or should) find the shareholder liable for the subsidiary's obligations. On occasion, joinder might be justified on the basis of consent, as when a parent agrees to be bound in an arbitration based on contracts signed by its subsidiary. Even though both entities have agreed to subject themselves to the same arbitral proceeding, the arbitrator might determine that neither company is liable for the other's obligations (Park, 2009, p.6). 
del velo; es decir, estableciendo que se ha concretado con solo aplicar la extensión del convenio, cualquier situación en donde el árbitro se declare competente será ya una aplicación de la doctrina (independientemente del resultado de la decisión del tribunal arbitral).

Ahora bien, una extensión del convenio sin aplicación del remedio jurídico, a la luz de la visión societaria, se consideraría una extensión concreta; debido a que el levantamiento del velo es, en sí mismo, un remedio jurídico para establecer la solución al problema del fraude. Es importante recordar que la extensión es un paso necesario para que los árbitros puedan aplicar el remedio del levantamiento de velo societario. De hecho, tal aspecto es el fin último de la extensión: abrir la posibilidad a los árbitros de aplicar (o no) el remedio. Por lo tanto, contar solo con el primer paso (y no con la consecuencia) sin la ruptura de la responsabilidad limitada no justifica, para la doctrina societaria, seguir utilizando la nomenclatura del levantamiento del velo societario.

\section{E. Sobre la poca conveniencia de crear un le- vantamiento del velo jurisdiccional}

Respecto a la posibilidad de un levantamiento del velo jurisdiccional, la decisión de competencia del tribunal arbitral depende del análisis de indicios que cumplen con satisfacer los requisitos necesarios para la extensión; y, como los elementos objetivos y subjetivos para la extensión del convenio son analizar la conformación del grupo económico y la voluntad de fraude, estos elementos tendrán que ser necesariamente analizados en la decisión de fondo de la controversia. Por tal motivo, ¿se puede hablar del levantamiento del velo jurisdiccional?

La competencia del tribunal dependerá de la identificación de diversos indicios que otorgan a los árbitros la apariencia de una alta probabilidad de responsabilidad. Este aspecto genera que la línea entre el análisis de competencia y el análisis de fondo sea sumamente borrosa. En efecto, crear la nomenclatura del levantamiento del velo jurisdiccional para referirse a la causa de la extensión del convenio arbitral solo generaría más confusiones con respecto a la doctrina societaria (riesgo que se ve reducido si observamos la extensión del convenio como parte de un todo), una respuesta la necesidad de actuar contra el fraude a los acreedores.

Por lo tanto resulta más conveniente construir, en el arbitraje, la abstracción jurídica de un procedimiento que tiene como paso principal y necesario la extensión del convenio arbitral. Además, su primordial consecuencia es la aplicación del remedio; es decir, la asignación de responsabilidad al tercero no firmante. Ahora bien, al ser la doctrina del levantamiento societario un supuesto de extensión del convenio tan peculiar (cuyo principal objetivo es el análisis de los factores que sirven para declarar la competencia del tribunal y para asignar responsabilidad) no es necesario hacer un corte y establecer una suerte de dos levantamientos del velo. Más bien un primer paso será construir un estándar de razonabilidad suficiente y no exagerado, el cual, al ser superado, justifique la existencia del riesgo de fraude.

Este primer aspecto tiene como consecuencia la extensión del convenio arbitral y la incorporación de la matriz o accionista al arbitraje. El segundo, asimismo, se refiere al análisis de todos los factores en el caso concreto; no solo para confirmar las conclusiones que permitieron superar el primer estándar de razonabilidad, sino para que, luego de la actuación de todas las pruebas, los árbitros concluyan que corresponde levantar el velo societario y responsabilizar a los no signatarios por el perjuicio a los acreedores.

¿Qué ocurriría si el segundo aspecto no concluye con la asignación de responsabilidad? En ese caso, se logró extender el alcance de la cláusula arbitral para que el análisis de responsabilidad se realice frente a una parte no signataria; sin embargo, el remedio jurídico de ignorar la responsabilidad limitada no fue utilizado.

\section{F. Hacia la construcción del nuevo paradigma del levantamiento del velo societario como extensión del convenio arbitral}

El problema planteado al inicio del presente análisis se centró en que no se puede determinar que al demandado no signatario se le debe extender el convenio arbitral sin antes (o a su vez) asignarle responsabilidad en tanto que, para que se configure la extensión del convenio por la teoría del levantamiento del velo, se requiere probar (por lo menos) un fraude o propósito indebido.

Debido a la inevitable conexión entre el análisis de fondo con respecto al análisis de jurisdicción, no es suficiente evaluar a la consecuencia (remedio jurídico), sino también al cuidadoso proceso que se sigue a fin de concluir la asignación de responsabilidad. Se podrá hablar de una relación de causalidad entre la perspectiva de la extensión y la del remedio cuando se identifican dos aspectos en concreto. Es decir, tanto en lo referente a los requisitos para la extensión del convenio arbitral, en los cuales intervienen no signatarios en el proceso como a la asignación de responsabilidad a los terceros no signatarios luego de haber analizado 
los indicios suficientes para comprobar la voluntad de fraude. Asimismo, que no exista ninguna otra herramienta que pueda compensar el daño hacia los acreedores (elemento de residualidad).

Sin embargo, en caso se hayan cumplido los requisitos para la extensión del convenio arbitral, el tribunal arbitral concluyó que la matriz o accionista no signatario no responde por el incumplimiento. El supuesto no calificaría como el levantamiento del velo societario ya que no se "rasgó" el velo societario para que un nuevo deudor responda por las acreencias de la compañía signataria ${ }^{13}$. En conclusión, si se mantiene la responsabilidad limitada, se mantiene el velo como remedio.

Si bien el tribunal arbitral puede emitir un laudo parcial para resolver las excepciones de jurisdicción en cualquier etapa del arbitraje, pareciese que la complejidad del análisis sobre el levantamiento del velo dificulta que una decisión sobre la competencia del tribunal sea brindada en un momento diferente al laudo final. Esta característica se genera a causa de la relevancia fundamental de las pruebas para generar convicción en el tribunal sobre la extensión del pacto arbitral al no signatario.

Ahora bien, ¿existen casos en que el tribunal arbitral puede resolver las cuestiones jurisdiccionales sobre levantamiento del velo en un momento diferente al laudo final? Consideramos que sí. El tribunal arbitral, en cada caso concreto, es el encargado de determinar si se han cumplido o no con los supuestos de extensión del convenio arbitral a una parte no signataria. En el caso de la doctrina del levantamiento del velo dichos supuestos están ligados a la verificación del fraude por los árbitros. Por ello, la concepción pareciera evidenciar una aparente mezcla entre el análisis de jurisdicción y de fondo. Sin embargo, podrían generarse malinterpretaciones sobre si el tribunal arbitral primero asignó responsabilidad y luego extendió el convenio (o viceversa).

Para evitar cualquier tipo de controversia como la anterior, debe quedar claro que la decisión sobre la extensión del pacto no sujeta o limita a los árbitros en su análisis de fondo. Un tribunal podría evaluar decidir sobre las cuestiones de jurisdicción en un laudo parcial y luego emitir un laudo final sobre los otros aspectos controvertidos referidos a la responsabilidad y cuantía de los daños. Por ejemplo, luego de haber establecidos indicios determinantes de fraude se podría responsabilizar a los demandados por una pretensión que establece daño patrimonial, pero declararse infundada la pretensión que solicita el pago de daños extrapatrimoniales.

Finalmente, es importante buscar una nueva forma de abordar el análisis de levantamiento del velo societario como supuesto de extensión del convenio arbitral. Un punto de partida podría ser dejar de considerar necesario el descubrimiento de un elemento que brinde una "certeza" o "apariencia" de fraude, de tal forma que sea suficiente la sola comprobación de una unidad económica entre la parte demandada y los no signatarios. Este último aspecto complementa los criterios establecidos por el artículo 14 de la Ley de Arbitraje. Dichos complementos serían los siguientes:

- Consentimiento determinado a partir de la buena fe.

- Comprobación de participación activa y determinante en la negociación, celebración, ejecución o terminación del contrato.

- $\quad$ La pretensión de derivar derechos o beneficios del contrato.

A partir de ello, un nuevo paradigma será dejar de mantener los elementos de análisis de fraude en la controversia sobre la jurisdicción o, por lo menos, reducirlos a un estándar de razonabilidad que sea acorde a la consecuencia. Es decir, se aplicaría la extensión del convenio, pero no se debería analizar dicha incorporación como si fuera una suerte de asignación adelantada de responsabilidad.

En ese sentido, el tribunal tendrá mayor libertad para poder decidir sobre su competencia en una etapa temprana a fin de evitar que los laudos sean materia de anulación, en tanto que el análisis (independiente del levantamiento del velo como remedio) implicaría un adelantamiento de opinión sobre el fondo de la controversia y la afectación al derecho de defensa de los no signatarios o demandados.

\section{REFLEXIONES FINALES}

A partir del análisis de las figuras del levantamiento del velo societario como remedio sustancial y presupuesto de extensión del pacto arbitral, podemos concluir lo siguiente:

13 Es importante precisar que, para los efectos del presente artículo, ignorar el límite de responsabilidad limitada de una compañía para poder alcanzar a la otra (que solo funciona para el elemento de asignación de responsabilidad) y el análisis de responsabilidad de daños conforman el llamado "análisis de fondo de la controversia". 
a) El levantamiento del velo como remedio sustancial, independientemente de la jurisdicción en que se aplique, se da como consecuencia de descubrir actos fraudulentos que llevan a cuestionar la responsabilidad limitada entre compañías. La causa puede ser diversa dependiendo las teorías que cortes judiciales o tribunales han establecido para analizar indicios y cuya consecuencia es buscar que los créditos sean satisfechos por la persona (natural o jurídica) que realmente controlaba las decisiones fraudulentas y no por la compañía que actuó como fachada.

b) En el levantamiento del velo como remedio originalmente judicial, la competencia de los jueces se desarrolla a partir del propio remedio. Es decir, cuando inicia el juicio la parte demandante abre proceso a la compañía y a sus accionistas o matrices. Es en el propio juicio que se analizan dos aspectos: (i) la unidad entre todos los involucrados; y, (ii) si el actuar constituye un acto fraudulento.

c) El levantamiento del velo como extensión del convenio arbitral se aplica puesto que, en el arbitraje, existe la necesidad de otorgarle competencia al árbitro a fin que pueda manifestarse sobre una controversia que incluiría partes no signatarias. La peculiaridad de dicha doctrina consiste en que el análisis de jurisdicción y fondo de la controversia tienen casi los mismos factores a analizar, pero con diferentes consecuencias. En efecto, el primero de ellos extiende el convenio arbitral a partes no signatarias, mientras que el segundo asigna responsabilidad a la parte no firmante.

d) No obstante, el objetivo de ambas figuras jurídicas es el mismo: la necesidad de evitar utilizar la no suscripción del contrato o la responsabilidad limitada en una compañía como excusa para la defraudación de acreedores. Con este aspecto en mente, se busca asignarles responsabilidad mediante la flexibilización de figuras rígidas que pudiesen impedir una verdadera justicia.

e) La referencia terminológica al levantamiento del velo jurisdiccional como consecuencia de la extensión del convenio arbitral a un no signatario genera confusiones sobre el verdadero entendimiento de la figura, la cual fue originariamente concebida como remedio judicial. Esta confusión puede reducirse si empezamos a observar la extensión del convenio como parte de un todo, lo cual configuraría una respuesta a la necesidad de actuar contra el fraude a los acreedores. f) La doctrina del levantamiento del velo societario necesita evolucionar e ir más allá de la terminología para responder a las nuevas necesidades jurisdiccionales en el arbitraje. Dicha doctrina debe tener una perspectiva ampliada y construirse como un procedimiento que no debe aislarse en dos levantamientos separados del velo trabajado.

Con las conclusiones presentes, se procederá a desarrollar, de forma breve, nuestra propuesta sobre el estado en cuestión.

Al establecer como un nuevo paradigma del levantamiento del velo societario como extensión del convenio, el dejar mantener los elementos de análisis de fraude en la controversia sobre la jurisdicción o, al menos, reducirlos a un estándar de razonabilidad que sea acorde a la consecuencia; el tribunal tendrá mayor libertad para poder decidir sobre su competencia en una etapa temprana del proceso. En ese sentido, la propuesta establece que la herramienta de la extensión no conserve los elementos de apariencia de fraude o, en caso sean estrictamente necesarios, que se restrinjan a un superficial análisis de conductas sospechosas.

En efecto, no es necesario que el tribunal arbitral concentre todo su esfuerzo en determinar, a partir de las pruebas, factores para comprobar la alta probabilidad de fraude en el grupo económico. Asimismo, tampoco se debe pretender hallar un indicio determinante de actos fraudulentos, puesto que no es necesario para cumplir con los requisitos que establece el artículo 14 de la Ley de Arbitraje. La conclusión de dicha herramienta solo es la extensión del convenio arbitral a partes no signatarias y, por ello, no es la asignación de responsabilidad.

El análisis de fondo de la controversia (la clara intención de defraudación) si deberá buscar indicios determinantes de fraude; a fin de justificar la aplicación del remedio jurídico, siempre y cuando se mantenga el principio de residualidad. Existirá una relación de causalidad entre ambas figuras cuando el análisis de fondo asigne responsabilidad a las partes no signatarias, pero no debería ocurrir que la justificación de la herramienta de extensión del convenio agote o pretenda agotar el análisis de fondo.

En ese sentido, para evitar cualquier tipo de controversia, debe ser claro que la decisión sobre la extensión del pacto no sujeta o limita a los árbitros en su análisis de fondo. Una opción sería que el tribunal evalúe decidir sobre las cuestiones de jurisdicción en un laudo parcial y luego emitir un laudo final sobre los otros aspectos controvertidos referidos a la responsabilidad y cuantía de los daños. ${ }^{{ }^{\circ} \mathbf{l}^{\circ}}$ 


\section{REFERENCIAS}

Besson, S. (2010). Piercing the corporate veil: back on the right track. En B. Hanotiau \& E. Schwartz (Eds.). Dossier VII of the ICC Institute of World Business Law: Multiparty Arbitration (pp. 147-161). International Chamber of Commerce Publication.

Born, G. (2009). International commercial arbitration (Vol. 1). Kluwer Law International.

(2014). Parties to International Arbitration Agreements. International Commercial Arbitration (2da. ed., Vol.1). Kluwer Law International.

Bullard González, A. (2008). Extensión del convenio arbitral. En A. Bullard González \& C. Soto Coaguila (Coords.). Comentarios a la Ley Peruana de Arbitraje (pp. 226-ss.). Instituto Peruano de Arbitraje.

Caivano, R. (2006). Arbitraje y grupos de sociedades. Extensión de los efectos de un acuerdo arbitral a quien no ha sido signatario. Lima Arbitration, (1), 121-162.

http://www.limaarbitration.net/LAR1/roque j_caivano.pdf

Cheng-Han, T., Wang, J., \& Hofmann, C. (2019). Piercing the Corporate Veil: Historical, Theoretical and Comparative Perspectives. Berkeley Business Law Journal, (16), 140-204. https://doi.org/10.2139/ssrn.3254130

Cohen, M. (2008). Grounds for Disregarding the Corporate Entity and Piercing the Corporate Veil. American Jurisprudence Proof of Facts. Thomson West.

Conejero Roos, C. \& Irra de la Cruz, R. (2012). La extensión del acuerdo arbitral a partes no signatarias en la Ley de Arbitraje peruana: algunas lecciones del derecho comparado. Lima Arbitration, (5), 56-91.

http://limaarbitration.net/LAR5/Cristian_Conejero_Roos_Rene_Irra_de_la_Cruz.pdf

De Trazegnies Granda, F. (2004). El rasgado del velo societario dentro del arbitraje. Ius et veritas, (29), 12-22.

Díaz-Candia, H. (2015). Tendencias actuales del arbitraje en Latinoamérica. Arbitraje: revista de arbitraje comercial y de inversiones, 8(2), 413-442.

Ferrario, P. (2009). The Group of Companies Doctrine in International Commercial Arbitration: Is There any Reason for this Doctri- ne to Exist? Journal of International Arbitration, 26(5), 647-673.

Garcia-Gallont, R., \& Kilpinen, A. J. (2015). If the Veil Doesn't Fit ... An Empirical Study of 30 Years of Piercing the Corporate Veil in the Age of the LLC. Wake Forest Law Review, 50(5), 1229-1252.

https://www.masader.om/eds/detail?db=bsu \&an $=116240000$ \&isbn $=0043003 \mathrm{X}$

Hansmann, H., \& Kraakman, R. (2003). El rol esencial del derecho en las organizaciones. THEMISRevista de Derecho, (46), 15-45.

http://revistas.pucp.edu.pe/index.php/themis/ article/view/9963 /10378

Macey, J., \& Mitts, J. (2014). Finding Order in the Morass: The Three Real Justifications for Piercing the Corporate Veil. Cornell Law Review, 100(1), 99-154.

https://scholarship.law.cornell.edu/cgi/viewcontent.cgi ?article $=4647 \&$ context $=c l r$

Palacios Pareja, E. (2008). El levantamiento del velo societario en el proceso arbitral. Advocatus, (18), 167-173.

https://doi.org/10.26439/advocatus2008. n018.2973

Park, W. (2009). Non-Signatories and International Contracts: An Arbitrator's Dilemma, edited by the Permanent Court of Arbitration. Oxford University Press.

Repetto, J. L., Hundskopf, A., \& Valderrama, M. (2014). Mi otro yo: La doctrina del alter ego y el artículo 14 de la Ley peruana de Arbitraje. Forseti, Revista de derecho, 2(2), 206-226.

http://forseti.pe/revista/arbitraje-internacional/ articulo/mi-otro-yo-la-doctrina-del-alter-ego-yel-articulo-14-de-la-ley-peruana-de-arbitraje

\section{LEGISLACIÓN, JURISPRUDENCIA Y OTROS DOCU-} MENTOS LEGALES

Primera Sala Civil con Subespecialidad Comercial de la Corte Superior de Justicia de Lima, Sentencia recaída en el expediente 451-2009, de 5 de marzo de 2013 (Perú).

Decreto Legislativo 1071. Decreto Legislativo que norma el Arbitraje. Diario Oficial El Peruano, 1 de septiembre de 2008 (Perú).

Salomon c. A Salomon \& Co Ltd. [1896] U.K.H.L. 1 (HL), [1897] A.C. 22 (Reino Unido).

Van Dorn Co. v. Future Chemical and Oil Corp., 753 F.2d 565 (7mo Cir. 1985) (EE. UU.).

Walkovszky c. Carlton, 223 N.E.2d 6 (N.Y. 1966) (EE. UU.). 